\title{
Safranal Treatment Improves Hyperglycemia, Hyperlipidemia and Oxidative Stress in Streptozotocin-Induced Diabetic Rats
}

\author{
Saeed Samarghandian ${ }^{1,2}$, Abasalt Borji ${ }^{1,2}$, Mohammad Bagher Delkhosh ${ }^{2}$, Fariborz Samini ${ }^{3}$ \\ ${ }^{1}$ Department of Basic Medical Sciences, Neyshabur University of Medical Sciences, Neyshabur, Iran. ${ }^{2}$ Health Strategic \\ Research Center, Neyshabur University of Medical Sciences, Neyshabur, Iran. ${ }^{3}$ Neurosurgery Department, Faculty of \\ Science, Mashhad University of Medical Sciences, Mashhad, Iran
}

Received, March 8, 2013; Accepted, July 24, 2013; Published, August 2, 2013.

\begin{abstract}
Purpose. Clinical research has confirmed the efficacy of several plant extracts in the modulation of oxidative stress associated with diabetes mellitus. Findings indicate that safranal has antioxidant properties. The aim of the present study was the evaluation of possible protective effects of safranal against oxidative damage in diabetic rats. Methods. In this study, the rats were divided into the following groups of 8 animals each: control, untreated diabetic, three safranal $(0.25,0.50,0.75 \mathrm{mg} / \mathrm{kg} / \mathrm{day})$-treated diabetic groups. Diabetes was induced by streptozotocin (STZ) in rats. STZ was injected intraperitoneally at a single dose of $60 \mathrm{mg} / \mathrm{kg}$ for diabetes induction. Safranal (intraperitoneal injection) was administered 3 days after STZ administration; these injections were continued to the end of the study (4 weeks). At the end of the 4-week period, blood was drawn for biochemical assays. In order to determine the changes of cellular antioxidant defense systems, antioxidant enzymes including glutathione peroxidase (GSHPx), superoxide dismutase (SOD) and catalase (CAT) activities were measured in serum. Moreover we also measured serum nitric oxide (NO) and serum malondialdehyde (MDA) levels, a marker of lipid peroxidation. Results. STZ-induced diabetes caused an elevation $(p<0.001)$ of blood glucose, MDA, NO, total lipids, triglycerides and cholesterol, with reduction of GSH level and CAT and SOD activities. The results indicated that the significant elevation in the blood glucose, MDA, NO, total lipids, triglycerides, cholesterol and reduction of glutathione level and CAT and SOD activity were ameliorated in the safranal-treated diabetic groups compared with the untreated groups, in a dose dependent manner $(p<0.05$, $p<0.01, p<0.001)$. Conclusion. These results suggest that safranal has antioxidant properties and improves chemically-induced diabetes and its complications by modulation of oxidative stress.
\end{abstract}

This article is open to POST-PUBLICATION REVIEW. Registered readers (see "For Readers") may comment by clicking on ABSTRACT on the issue's contents page.

\section{INTRODUCTION}

Diabetes mellitus is a metabolic disease as old as mankind and its incidence is considered to be high $(4-5 \%)$ all over the world. It is defined as a group of disorders characterized by hyperglycemia and altered metabolism of lipids, carbohydrates and proteins. Diabetes mellitus is the principal factor responsible for renal failure, blindness and nontraumatic amputations; the connection between diabetes with poor metabolic control and the high prevalence of mortality due to coronary heart disease, retinopathy, nephropathies and neuropathies has been well established. Furthermore, diabetes mellitus in turn leads to hyperlipidemia to cardiovascular morbidity and mortality. Nevertheless, control of plasma glucose and lipid concentrations inhibit micro-vascular complications. For these considerations the main objective of medical treatment in patients with diabetes is metabolic control (1).

Oxidative stress results from an imbalance between radical-generating and radical-scavenging systems, i.e. increased free radical production or reduced activity of antioxidant defenses or both (2). Implication of oxidative stress in the pathogenesis of diabetes is suggested, not only by oxygen freeradical generation, but also due to nonenzymatic protein glycosylation, auto-oxidation of glucose,

Corresponding Author: Saeed Samarghandian, Department of Basic Medical Sciences, Neyshabur University of Medical Sciences, Neyshabur, Iran; Email:samarghandians@mums.ac.ir 
impaired glutathione metabolism, alteration in antioxidant enzymes and lipid peroxides formation (3). In addition to glutathione (GSH), there are other defense mechanisms against free radicals, including the enzymes superoxide dismutase (SOD), glutathione peroxidase (GPx) and catalase (CAT) whose activities contribute to elimination of superoxide, hydrogen peroxide and hydroxyl radicals (4-8).

However, evidence that antioxidants can prevent cells against the cytotoxic effects of free radicals is still relatively scarce. In the last decade, much attention was focused on the biological and medical properties of an ancient spice, saffron and its ingredients (9). Recent scientific findings have been encouraging, uniformly showing that saffron and its component (safranal) can affect carcinogenesis and have currently been studied extensively as a most promising cancer chemopreventive agent $(10,11)$. Furthermore safranal could be a candidate to suppress the development of age-induced damage by protecting against oxidative stress and by increasing antioxidant defenses (12). Different hypotheses for the modes of anticarcinogenic and antitumor actions of saffron and its components have been proposed $(13,14)$; one of them is the inhibitory effect on free radical chain reactions. In fact, safranal is lipidsoluble and might act as a membrane-associated high-efficiency free radical scavenger, which is connected with its antioxidant properties (15). This study focuses on properties of safranal that significantly decreased the levels of free radicals and act as an antioxidant. Also, in modern pharmacological studies, saffron, or its active constituents, has demonstrated beneficial effects in reducing or preventing tissue damages in pathological conditions (16). Recently, it was shown that saffron extract, crocin and safranal also had significant radical scavenging activities and consequent antioxidant activity (17). These studies indicated that safranal was a potent antioxidant and was able to protect body organs against toxic materials in diabetes. However, there are no reports about the possible mechanisms and clinical uses of safranal in diabetes mellitus and much more basic pharmacological and toxicological studies are needed to support clinical trials to evaluate the safety, tolerability and efficacy of safranal. Thus, the present study was designed to evaluate the effect of safranal on a model of diabetes mellitus and its effects on serum lipid profiles and oxidative stress parameters in this process.

\section{MATERIALS AND METHODS}

\section{Reagents}

All purified enzymes, coenzymes, substrates, standards, buffers, kits and also safranal and other chemicals were purchased from Sigma-Aldrich Chemical (St. Louis, USA).

\section{Animals}

Wistar albino rats (2 months; $200 \pm 13$ g) were bred at the university experimental animal care centre. Animals were maintained under standard environmental conditions and had free access to standard rodent feed and water

\section{Study Design}

45 male Wistar albino rats were randomly allotted to five experimental groups ( $\mathrm{n}=8$ per group) as follows: group 1, control (C); group 2, diabetic (D); group 3, diabetic and safranal-treated $(0.25$ $\mathrm{mg} / \mathrm{kg} /$ day) (D + S1); group 4, diabetic and safranal-treated $(0.5 \mathrm{mg} / \mathrm{kg} /$ day $)(\mathrm{D}+\mathrm{S} 2)$; group 5 , diabetic with safranal-treated $(0.75 \mathrm{mg} / \mathrm{kg} /$ day $)$ (D + S3). Rats were kept in their own cages at constant room temperature $\left(21 \pm 2{ }^{\circ} \mathrm{C}\right)$ under a normal 12-h light/dark cycle with free access to food and water. The animals were housed according to regulations for the Welfare of experimented animals. The study was conducted in Mashhad Medical University Experimental Animal Research Laboratory. Protocols were approved by the Ethical Committee (The Ethical Research Committee of Mashhad University of Medical Sciences). On the first day of the study, the diabetic groups were given streptozocin in a single intraperitoneal (i.p.) injection at a dose of $60 \mathrm{mg} / \mathrm{kg}$ for induction of diabetes. Blood was extracted from the tail vein for glucose analysis 72 hours after streptozocin injection. Rats with blood glucose levels higher than $250 \mathrm{mg} / \mathrm{dl}$ were accepted as being diabetic. In the control groups (C), physiological saline (i.p.) was injected as vehicle. Safranal (i.p. injection) was administered to the treatment groups from 3 days after STZ administration; these injections were continued to the end of the study (for 4 weeks). Blood glucose level and body weights were recorded at weekly intervals. At the end of the 4week period, animals were killed by pentobarbital 
overdose (150 mg/kg, i.p.), and blood was subsequently collected from the retro orbital sinus. Blood and sera were separated by centrifugation at $3000 \mathrm{rpm}$ for $10 \mathrm{~min}$ for glucose, lipid profile, malondialdehyde (MDA), GSH, CAT, SOD and nitric oxide (NO) assays.

\section{Measurement of Blood Glucose}

Glucose concentrations were measured with the Ames One Touch glucometer (One-Touch Basic; Lifescan, Johnson and Johnson, New Brunswick, $\mathrm{NJ}$ ) in rat tail vein blood. Blood glucose was estimated using the diagnostic kits (Pars Azmoon kit, IRI) on an automatic analyzer (Abbott, model Alcyon 300, USA).

\section{Measurement of Serum Lipid Profile}

The concentrations of glucose, total lipids, triglycerides, total cholesterol, low-density lipoprotein (LDL) cholesterol and high-density lipoprotein (HDL) cholesterol in serum were estimated by using diagnostic kits (Pars Azmoon kit, IRI) on an automatic analyzer (Abbott, model Alcyon 300, USA)

\section{Measurement of Serum Reduced Glutathione (GSH)}

GSH was determined by the method of Ellman (1959). To the homogenate was added $10 \%$ of trichloracetic acid (TCA) and centrifuged. $1.0 \mathrm{ml}$ of supernatant was treated with $0.5 \mathrm{ml}$ of Ellman's reagent (19.8 $\mathrm{mg}$ of 5, 5'-dithiobisnitro benzoic acid (DTNB) in $100 \mathrm{ml}$ of $0.1 \%$ sodium nitrate) and 3.0 $\mathrm{ml}$ of phosphate buffer $(0.2 \mathrm{M}, \mathrm{pH} 8.0)$. The absorbance was read at $412 \mathrm{~nm}$ (18).

\section{Measurement of Serum Thiobarbituric Acid Reactive Species (TBARS)}

The formation of lipid peroxides was measured in the serum. The formation of MDA, an end product of fatty acid peroxidation was measured spectrophotometrically at $532 \mathrm{~nm}$ by using a thiobarbituric acid reactive substance (TBARS), essentially by the method of Genet et al. (2002). Results are expressed as nmole of MDA formed/mg protein (19).

\section{Measurement of Serum Superoxide Dismutase (SOD) activity}

The activity of SOD was determined by a method using inhibition of pyrogallol autoxidation at $\mathrm{pH} 8$
(20). The specific activity of SOD is expressed as units per mg protein per minute.

\section{Measurement of Serum Catalase (CAT) Activity} Catalase activity was assayed by $\mathrm{H}_{2} \mathrm{O}_{2}$ consumption, following Aebi's(1984) method and modified by Pieper et al. (1995). Briefly, ethanol was added $(1: 100 \mathrm{v} / \mathrm{v})$ to the supernatants and incubated for $30 \mathrm{~min}$ in an ice bath. $1 \%$ Triton X$100(1: 10 \mathrm{v} / \mathrm{v})($ Sigma) was then added to the homogenates. This solution was placed in an ice bath for an additional $15 \mathrm{~min}$. $500 \mu \mathrm{l}$ of this solution was placed into a glass cuvette and $250 \mu 1$ of $30 \mathrm{mM} \mathrm{H} \mathrm{H}_{2} \mathrm{O}_{2}$ (Sigma) in phosphate buffer (50mM, pH 7.0) was then added to start the reaction. After $15 \mathrm{~s}$ the absorbance at $240 \mathrm{~nm}$ was read every $15 \mathrm{~s}$ for $45 \mathrm{~s}$. Catalase activity was expressed as $\mathrm{mmol} \mathrm{H}_{2} \mathrm{O}_{2} / \mathrm{min} / \mathrm{mg}$ protein. An enzyme unit was defined as the amount of enzyme that catalyzes the release of one $\mu \mathrm{mol}$ of $\mathrm{H}_{2} \mathrm{O}_{2}$ per min. Specific activity was calculated in terms of units per $\mathrm{mg}$ of protein. The assay was performed at $25^{\circ} \mathrm{C}(21,22)$.

\section{Measurement of Serum Nitric Oxide (NO)}

NO levels are determined spectrophotometrically by measuring the accumulation of its stable degradation products, nitrite and nitrate. The serum nitrite level was determined by the Griess reagent according to Hortelano et al. (1995). The Griess reagent, a mixture $(1: 1)$ of $1 \%$ sulfanilamide in $5 \%$ phosphoric acid and $0.1 \%$ 1-naphthyl ethylenediamine gives a red violent diazo color in the presence of nitrite. The color intensity was measured at $540 \mathrm{~nm}$. Results were expressed as $\mu \mathrm{mol} / \mathrm{l}$ using a $\mathrm{NaNO}_{2}$ calibration graph (23).

\section{Measurement of Serum Protein Content}

Protein content was determined by the method of Lowry et al. (1951), using bovine serum albumin (BSA) as a standard (24).

\section{STATISTICAL ANALYSIS}

All experiments were carried out at least in duplicate. Each group consisted of eight rats. Oneway analysis of variance (ANOVA) was performed and Tukey post hoc test was used for multiple comparisons. Statistical analyses were performed using the InStat 3.0 program. The results are expressed as mean \pm SEM. The results originated 
from analysis of serum. Linear correlation tests were also performed. Differences of $p<0.05$ were considered significant.

\section{RESULTS}

During the experimental period (4-week), there was a weight loss in untreated diabetic rats compared with normal healthy rats (control) $(p<0.001)$ (Table 1). However, at the end of the experimental treatment period there was an elevation in body weight of safranal $(0.5$ and $0.75 \mathrm{mg} / \mathrm{kg})$ - treated diabetic rats compared to untreated diabetic rats $(p<0.05$ and $p<0.01$, respectively), but the elevated body weight in the safranal $(0.25$ and $0.5 \mathrm{mg} / \mathrm{kg})$ treated diabetic groups was significantly lower than the control group $(p<0.01)$ (Table 1). At the highest safranal dose $(0.75 \mathrm{mg} / \mathrm{kg})$ there were no significant differences in body weight compared to control rats after the 4-week experimental period (Table 1). STZ-diabetic rats exhibited significant ( $p$ $<0.001)$ hyperglycemia compared to the control rats (Figure 1). After 4 weeks the safranal dosedependently decreased blood glucose levels in the diabetic rats compared to the untreated diabetic rats (Figure 1). Safranal $(0.25 \mathrm{mg} / \mathrm{kg} /$ day) significantly decreased glucose in STZ diabetic rats only at the 4 th week of the study $(p<0.05)$, while at 0.5 $\mathrm{mg} / \mathrm{kg} /$ day safranal reduced blood glucose significantly at the $3 \mathrm{rd}$ and 4 th week from induction of diabetes compared with untreated diabetic rats $(p<0.05$ and $p<0.01$, respectively). At the highest dose of safranal $(0.75 \mathrm{mg} / \mathrm{kg} /$ day $)$ blood glucose of diabetic rats was significantly reduced beginning from the first week of treatment $(p<0.05, p<0.01$, $p<0.001$ ) (Figure 1).

STZ-injected rats showed significant increases in the serum levels of total lipids, triglycerides, total cholesterol and LDL-cholesterol (LDL-C), and significantly decreased serum HDL-cholesterol (HDL-C) level compared to the control group (Figure 2). Safranol dose-dependently reduced the

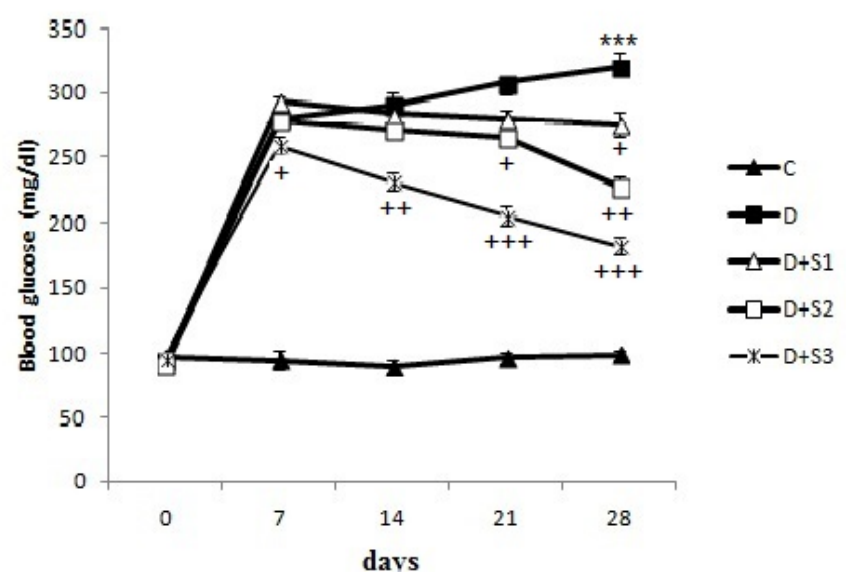

Figure 1. Effect of safranal on Blood Glucose Level $(\mathrm{mg} / \mathrm{dl})$. Control (C), untreated diabetic rats (D), safranal $(0.25 \mathrm{mg} / \mathrm{kg} /$ day $)$ - treated diabetic $(\mathrm{D}+\mathrm{S} 1)$, safranal $(0.5 \mathrm{mg} / \mathrm{kg} /$ day $)$ - treated diabetic $(\mathrm{D}+\mathrm{S} 2)$ and safranal $(0.75 \mathrm{mg} / \mathrm{kg} /$ day $)$ - treated diabetic $(\mathrm{D}+\mathrm{S} 3)$ rats during 4 weeks of study ( $n=8$, for each group).Values are the mean \pm SEM. Statistical significance for the difference between the data of the control group vs other groups: $* * * ; p<0.001$. Statistical significance for the difference between the data of untreated diabetic group vs treated groups: + ; $p<0.05,++; p<0.01,+++; p<0.001$.

Table 1. Effect of Safranal on Body Weight in STZ-treated Diabetic Rats. Control (C), untreated diabetic rats (D), safranal $(0.25 \mathrm{mg} / \mathrm{kg} /$ day $)$ - treated diabetic rats $(\mathrm{D}+\mathrm{S} 1)$, safranal $(0.5 \mathrm{mg} / \mathrm{kg} / \mathrm{day})$ - treated diabetic rats $(\mathrm{D}+\mathrm{S} 2)$ and safranal $(0.75 \mathrm{mg} / \mathrm{kg} / \mathrm{day})$ - treated diabetic rats $(\mathrm{D}+\mathrm{S} 3)$ during 4 weeks of study

\begin{tabular}{ccccc}
\hline Days 28 & $\mathbf{0}$ & $\mathbf{7}$ & $\mathbf{1 4}$ & $\mathbf{2 1}$ \\
\hline $\begin{array}{c}\text { C Body weight (g) } \\
265 \pm 15\end{array}$ & $180 \pm 12$ & $218 \pm 10$ & $231 \pm 13$ & $245 \pm 14$ \\
$\begin{array}{c}\text { D Body weight (g) } \\
150 \pm 15^{* * *}\end{array}$ & $175 \pm 12$ & $171 \pm 12^{* * *}$ & $164 \pm 10^{* * *}$ & $158 \pm 10^{* * *}$ \\
$\begin{array}{c}\text { D+S1 Body weight } \\
(\mathrm{g}) 183 \pm 15^{* *}\end{array}$ & $178 \pm 10$ & $174 \pm 6^{* *}$ & $176 \pm 8^{* *}$ & $180 \pm 13^{* *}$ \\
$\begin{array}{c}\mathrm{D}+\mathrm{S} 2 \text { Body weight } \\
(\mathrm{g}) 200 \pm 15^{* *,+}\end{array}$ & $179 \pm 9$ & $183 \pm 9^{*}$ & $190 \pm 11^{*}$ & $192 \pm 9^{* *+}$ \\
$\begin{array}{c}\mathrm{D}+\mathrm{S} 3 \text { Body weight } \\
(\mathrm{g}) 219 \pm 15^{++}\end{array}$ & $182 \pm 15$ & $187 \pm 11$ & $200 \pm 14$ & $211 \pm 21^{+}$ \\
\hline
\end{tabular}

Each measurement was done at least in triplicate and the values are the means \pm SEM for eight rats in each group.

Significantly different from normal control (Group C) rats $\left({ }^{*} ; P<0.05,{ }^{* *} ; P<0.01,{ }^{* * *} ; P<0.001\right.$ ).

Significantly different from STZ-treated (Group D) rats $\left({ }^{+} ; P<0.05,{ }^{++} ; P<0.01\right)$ 

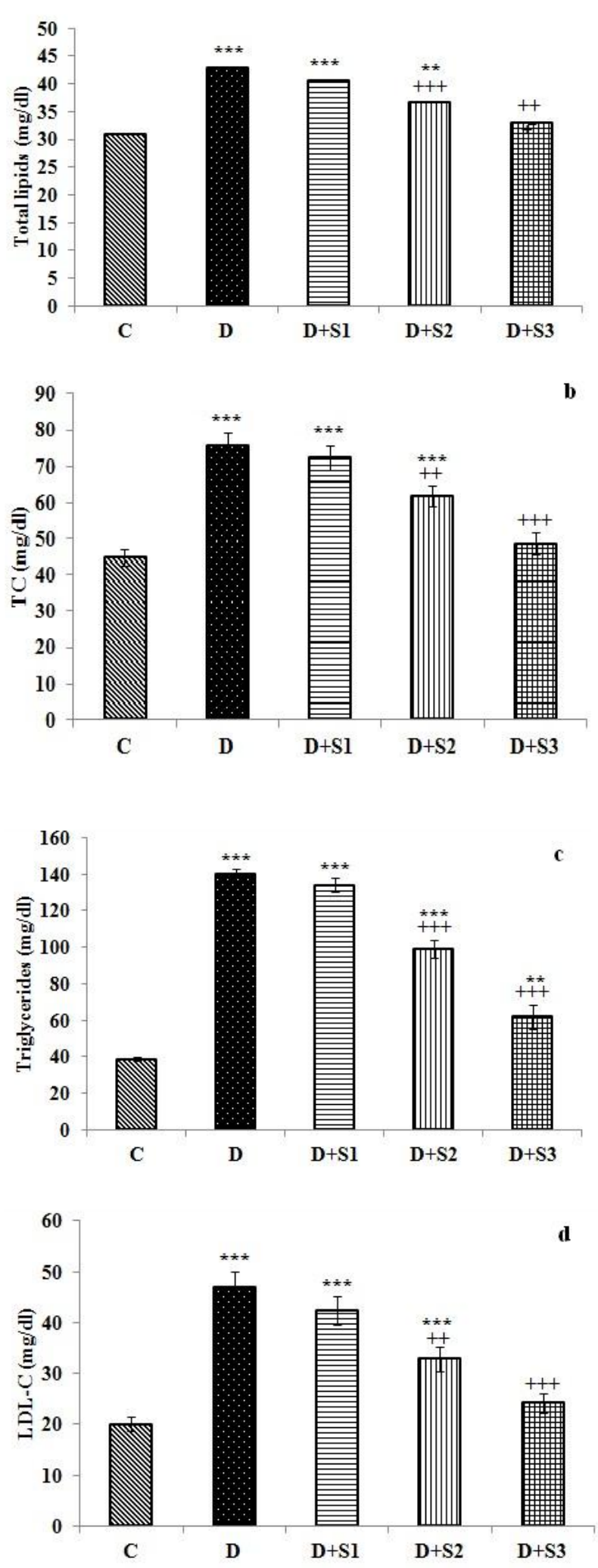

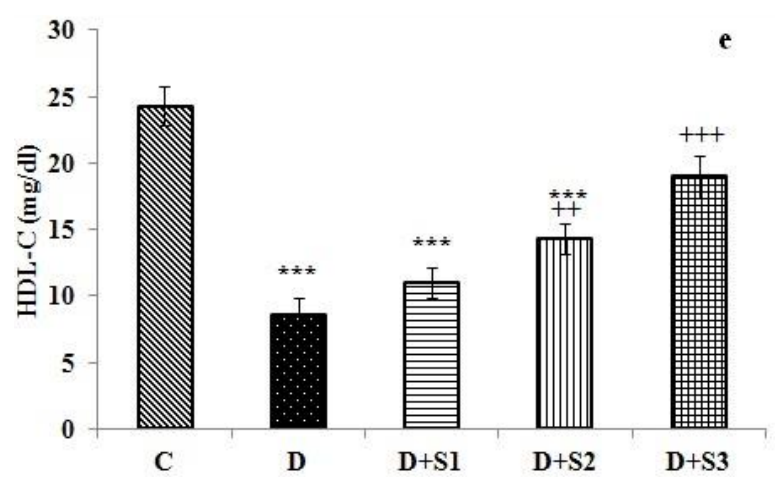

Figure 2. Effect of Safranal on Plasma Lipid Profiles (mg/dl). a: total lipid, b: total cholesterol (TC), c: triglycerides, d: LDL-C, e: HDL-C, in control (C), untreated diabetic rats (D), safranal $(0.25 \mathrm{mg} / \mathrm{kg} /$ day $)$ treated diabetic $(\mathrm{D}+\mathrm{S} 1)$, safranal $(0.5 \mathrm{mg} / \mathrm{kg} /$ day $)$ treated diabetic $(\mathrm{D}+\mathrm{S} 2)$ and safranal $(0.75 \mathrm{mg} / \mathrm{kg} /$ day $)$ treated diabetic $(\mathrm{D}+\mathrm{S} 3)$ rats during 4 weeks of study $(n=8$, for each group). Values are the mean \pm SEM. Statistical significance for the difference between the data of the control group vs other groups: **; $p<0.01$, $* * * ; p<0.001$. Statistical significance for the difference between the data of untreated diabetes group vs treated groups: $++; p<0.01,+++; p<0.001$.

serum levels of total lipids, triglycerides, total cholesterol and LDL-C, and increased serum HDL$\mathrm{C}$ level during the experimental period. At the highest safranal dose $(0.75 \mathrm{mg} / \mathrm{kg} /$ day $)$ there was no significant difference in total lipid, cholesterol, LDL-C and HDL-C levels between the STZ-treated rats and the control rats (Figure 2).

STZ injection produced significant changes in oxidative stress parameters in the serum of diabetic rats 4 weeks after diabetes induction, as shown by increased lipid peroxidation product (MDA) and decreased GSH compared to control group ( $p<$ 0.001) (Figure 3). Safranal (0.5 and 0.75 $\mathrm{mg} / \mathrm{kg} /$ day) significantly decreased the serum MDA and increased glutathione (GSH) compared with the untreated diabetic groups $(p<0.01$ and $p<0.001$, respectively).

The increase in GSH was dose-dependent, from $0.2 \pm 0.03$ at $0.5 \mathrm{mg} / \mathrm{kg}$ to $0.37 \pm 0.06$ at $0.75 \mathrm{mg} / \mathrm{kg}$ $(p<0.05)$. In addition, the MDA levels in animals which were administrated with high safranal concentration were significantly lower than the diabetic rats receiving low safranal concentration 
$(0.24 \pm 0.06$ vs $0.69 \pm 0.08, p<0.001)$ and MDA levels in diabetic rats treated with the medium concentration $(0.5 \mathrm{mg} / \mathrm{kg})$ were significantly lower than the low safranal treated diabetic rats $(0.43 \pm$ 0.03 vs $0.69 \pm 0.08, p<0.05)$. Furthermore, there was no significant difference between MDA and GSH levels between control rats and high dose safranal treated diabetic rats (Figure 3).
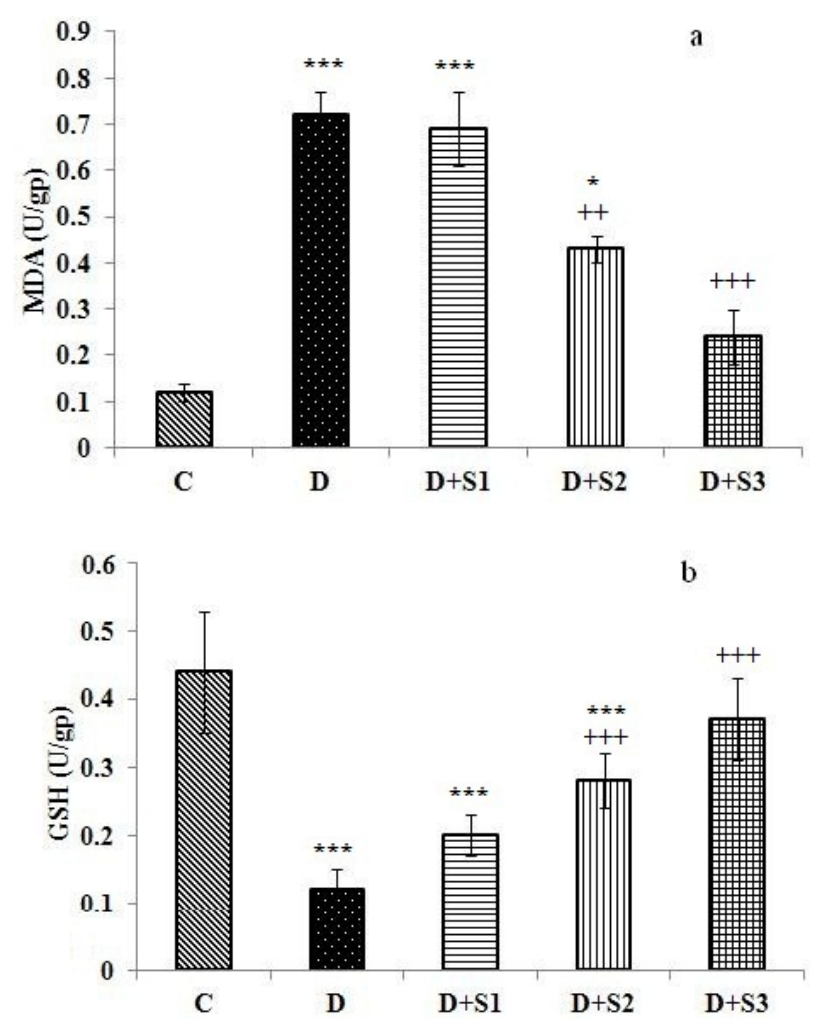

Figure 3. Effect of Safranal on MDA and GSH. Serum MDA (a) and GSH (b) levels (U/gp) in control (C), untreated diabetic rats (D), safranal $(0.25 \mathrm{mg} / \mathrm{kg} /$ day) treated diabetic $(\mathrm{D}+\mathrm{S} 1)$, safranal $(0.5 \mathrm{mg} / \mathrm{kg} /$ day $)$ treated diabetic $(\mathrm{D}+\mathrm{S} 2)$ and safranal $(0.75 \mathrm{mg} / \mathrm{kg} / \mathrm{day})$ treated diabetic $(\mathrm{D}+\mathrm{S} 3)$ rats during 4 weeks of study $(\mathrm{n}=8$, for each group).Values are the mean \pm SEM. Statistical significance for the difference between the data of the control group vs other groups: *; $p<0.05$, $* * * ; p<0.001$. Statistical significance for the difference between the data of untreated diabetes group vs treated groups: ++; $P<0.01$, +++; $P<0.001$.

SOD and CAT activities were decreased in the STZ-diabetic group compared with the control group $(p<0.001)$ (Figure 4). Safranal $(0.25,0.5$ and 0 . $75 \mathrm{mg} / \mathrm{kg} /$ day) treated diabetic rats had significantly increased serum SOD compared with the untreated diabetic rats $(p<0.05, p<0.001$ and $p<$ 0.001 , respectively). In addition, there was not a
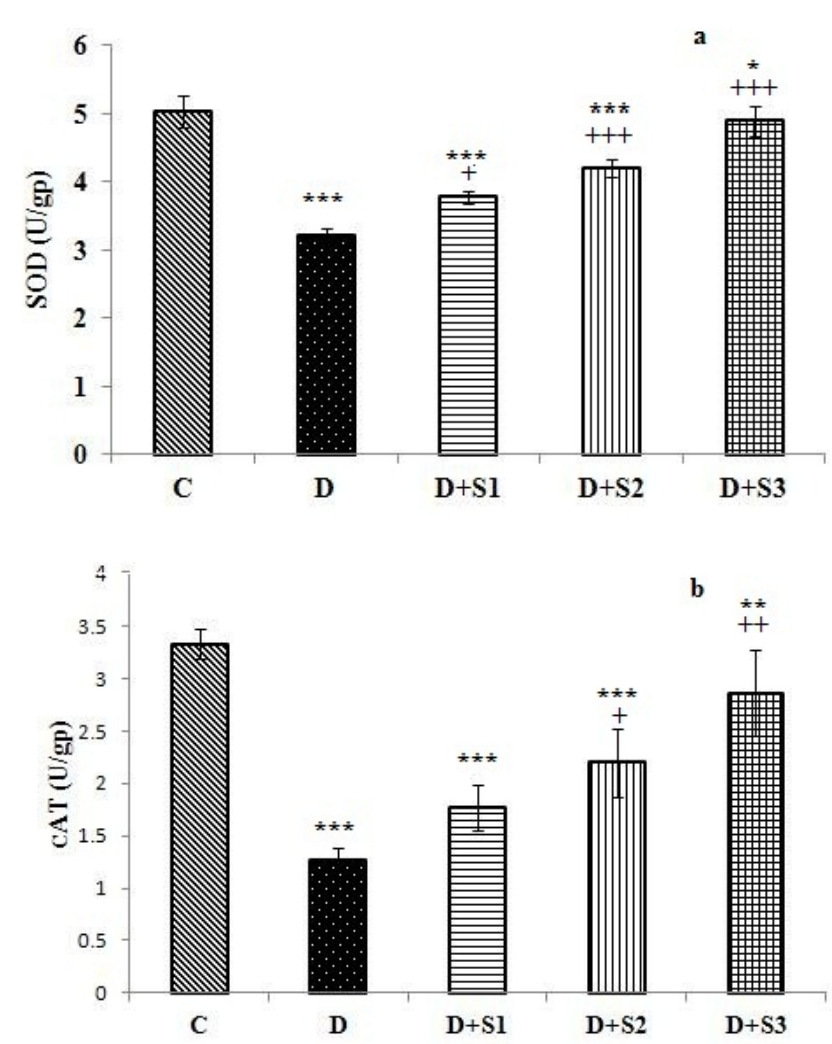

Figure 4. Effect of Safranal on Serum SOD (a) and CAT (b) $(\mathrm{U} / \mathrm{gp})$. Control (C), untreated diabetic rats (D), safranal $(0.25 \mathrm{mg} / \mathrm{kg} /$ day $)$ - treated diabetic $(\mathrm{D}+\mathrm{S} 1)$, safranal $(0.5 \mathrm{mg} / \mathrm{kg} /$ day $)$ - treated diabetic $(D+S 2)$ and safranal $(0.75 \mathrm{mg} / \mathrm{kg} /$ day $)$ - treated diabetic $(D+S 3)$ rats during 4 weeks of study ( $\mathrm{n}=8$, for each group). Values are the mean \pm SEM. Statistical significance for the difference between the data of control vs other groups: *; $p<0.05, * * ; p<0.01, * * * ; p<0.001$. Statistical significance for the difference between the data of diabetes $v$ s treated groups: +; $p<0.05,++; p<0.01$, +++; $p<0.001$.

significant difference between diabetic rats treated with high safranal concentration $(0.75 \mathrm{mg} / \mathrm{kg} / \mathrm{day})$ and the control group. The CAT activities in safranal $(0.5$ and $0.75 \mathrm{mg} / \mathrm{kg} /$ day $)$-treated diabetic rats were significantly higher than the untreated diabetic group $(p<0.05$ and $p<0.01$, respectively) (Figure 4). The CAT levels in animals which had been administrated high safranal dose were significantly greater than the diabetic rats receiving the low safranal dose $(2.86 \pm 0.41$ vs $1.77 \pm 0.22$, $\mathrm{P}<0.05)$. The effects on SOD and CAT were dosedependent, the activity of serum SOD in animals administrated with the high safranal dose $(0.75$ $\mathrm{mg} / \mathrm{kg}$ ) being significantly greater than that in the rats receiving low safranal concentration $(0.25$ 
$\mathrm{mg} / \mathrm{kg})(4.9 \pm 0.22$ vs $3.78 \pm 0.09, p<0.001) ;$ CAT activities in diabetic rats treated with medium concentration $(0.5 \mathrm{mg} / \mathrm{kg})$ were significantly higher than low safranal treated diabetic rats $(4.2 \pm 0.13 \mathrm{vs}$ $3.78 \pm 0.09, p<0.05$ ) (Figure 4).

STZ injection produced a significant increase of NO compared to the control group $(P<0.001)$ (Figure 5). Safranal $(0.25,0.5$ and $0.75 \mathrm{mg} / \mathrm{kg} /$ day) - treated diabetic rats had significantly decreased serum NO compared with the untreated diabetic rats $(p<0.001)$. The effects on NO were dosedependent, serum NO levels of animals having been administrated high safranal dose being significantly lower than those in the diabetic rats receiving the low safranal dose $(6.9 \pm 0.08$ vs $9.9 \pm 0.17$, $p<0.001)$; and NO levels in diabetic rats treated with a medium concentration $(0.5 \mathrm{mg} / \mathrm{kg})$ were significantly lower than the low safranal-treated diabetic rats $(8.5 \pm 0.21$ vs $9.9 \pm 0.17, p<0.001)$.

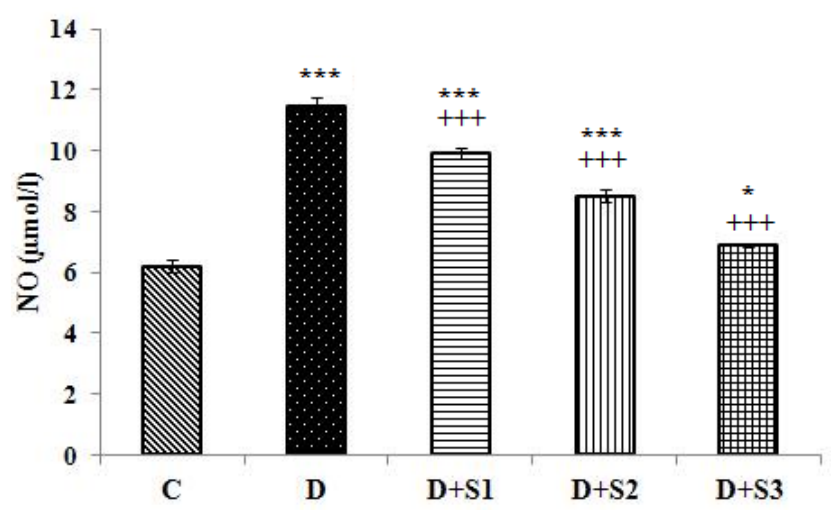

Figure 5. Effect of Safranal on Serum NO $(\mu \mathrm{M} / 1)$. Control (C), untreated diabetic (D), diabetic and $(0.25$ $\mathrm{mg} / \mathrm{kg} /$ day $)$ safranal $(0.25 \mathrm{mg} / \mathrm{kg} /$ day $)$ - treated diabetic $(\mathrm{D}+\mathrm{S} 1)$, safranal $(0.5 \mathrm{mg} / \mathrm{kg} /$ day $)$ - treated diabetic $(\mathrm{D}+$ S2) and safranal $(0.75 \mathrm{mg} / \mathrm{kg} /$ day $)$ - treated diabetic (D + S3) rats during 4 weeks of study $(n=8$, for each group).Values are the mean \pm SEM. Statistical significance for the difference between the data of control vs other groups: *; $p<0.05, * * * ; p<0.001$. Statistical significance for the difference between the data of untreated diabetes vs treated groups: +++ ; $p<0.001$.

\section{DISCUSSION}

The results of the present study indicate that intraperitoneal injection of safranal significantly ameliorated the adverse metabolic effects in rats treated with STZ.

Safranal injection after STZ treatment resulted in lower serum glucose levels, and improved lipid profile as well as body weight as compared with rats treated with STZ alone. In addition, safranal treatment of diabetic rats were observed to significantly recover the decreased levels of GSH, CAT and SOD, as well as the decline in the lipid profiles and the oxidative stress parameters MDA and NO compared with untreated diabetic group. These results are compatible with the findings reported by other investigators using saffron and its active constituent, crocin, which improved oxidative damage due to STZ and alloxan induced diabetes in rats (25-27). The effects observed in the present study are consistent with amelioration of oxidative stress in the STZ diabetic rats. The results are similar to the findings reported by other investigations using STZ to induce diabetes in rats, accompanied by an increase in the susceptibility to lipid peroxidation $(28,29)$.

Oxidative stress plays an important role in the pathogenesis and the complications of diabetes. Hyperglycemia results in overproduction of oxygen free radicals, which contributes to the progression of diabetes and its complications (28).

Several studies have shown that STZ produces imbalance between plasma oxidant and antioxidant content, resulting in the development of diabetes mellitus and its complications. STZ enters the $\beta$ cell via the low affinity glucose protein-2 transporter, inducing the selective destruction of the insulin producing islet $\beta$ cells and, in turn, a drastic reduction in insulin production. The cytotoxic effect of STZ could result from the combined action of DNA alkylation (30) and the cytotoxic effects of ROS (31) or the intracellular liberation of NO (32, 33) directly or indirectly through the formation of peroxynitrite. Thus, in the STZ-treated model of diabetes in rats, the diabetes that develops after the destruction of the pancreatic islets is associated strictly with the induction of oxidative and nitrosative stress, both systemically and locally. Furthermore, insulin resistance leads to adipocyte dysfunction and decrease in inhibition of the release of free acid into the plasma (34). The consequent increase in circulating free fatty acids (FFA) contributes to an increase in hepatic triglycerides. This increased hepatic triglyceride content contributes to an increase in the production of more 
atherogenic small dense LDL. These findings may constitute the predominant mechanism in STZinduced complications of hyperglycemia (35).

In our experimental model of diabetes mellitus, it was seen that STZ administration led to a significant decrease in plasma GSH content and CAT and SOD activities, accompanied by a significant increase in MDA, NO and overall lipid profile, indicating an increased plasma oxidative stress which may also occur in other tissues in STZtreated rats. MDA is an aldehydic product of lipid peroxidation that reacts quickly with biomolecules, such as proteins, lipids and nucleic acids and leads to cellular dysfunction, including liver and $\beta$ pancreatic cells, hence disturbing glucose regulation (36). The enhanced interaction of superoxide with NO in the oxidant environment of diabetes increases the formation of peroxynitrite, which in turn, oxidizes tetrahydrobiopterin (BH4, a cofactor for eNOS), leading to eNOS uncoupling (37). The diabetic condition favors the uncoupling of eNOS (38) and the increase in nitric oxide synthase (iNOS) expression (39), with a diminished NO bioavailability. Increasing evidence suggests that oxidative stress and changes in nitric oxide formation or action play major roles in the onset of diabetic complications such as atherosclerosis.

The improvement of variable measurements in STZ-diabetic rats after safranal treatment might suggest a protective influence of safranal against STZ action that could be mediated through suppression of oxygen free radicals induced by STZ. A stimulating effect of the formation of GSH by safranal was observed in the present study. The GSH reacts with free radicals and is a crucial substrate for glutathione peroxidase and glutathione-S-transferase, which take part in the cellular defense mechanisms against intermediate oxygen products $(40,41)$. It may be relevant that the ratio of GSH/GSSG plays a critical role in glucose homeostasis of diabetes because thiol groups are important in intracellular and membrane redox state (41) Safranal induced an increase in plasma GSH content, which might enhance the GSH/GSSG ratio and decrease lipid peroxidation, hence aldehydic concentration, and therefore improve serum glucose regulation. Parallel to these events, plasma CAT and SOD activity were increased in safranal treated diabetic rats as compared with untreated diabetic rats. SOD is responsible for removal of superoxide radicals and catalase decomposes hydrogen peroxide to water and oxygen; thus, these enzymes may contribute to the modulation of redox state of plasma (42). This observation perfectly agrees with those of Rahbani et al (2011) who demonstrated hypoglycemic and antioxidant activity of ethanolic saffron in STZinduced diabetic rats. Similarly, Kianbakht et al (2011) observed that saffron, crocin and safranal may effectively control glycemia in the alloxaninduced diabetes rat model $(16,26)$.

The results of the present study indicate that safranal is also effective to prevent hyperlipidemia due to diabetes. Safranal inhibits elevation of the serum lipid profile by controlling oxidative and nitrosative systems. Saffron has been reported to help lower cholesterol and keep cholesterol at healthy levels (43). In agreement with previous studies, one mechanism for the hypolipidemic effect of saffron extract and its constituents has proposed inhibitory effects on the levels of MDA, oxygen free radical and activating superoxide dismutase (44).

Reactive oxygen species are increased by hyperglycemia. Hyperglycemia, which occurs during diabetes (both type 1 and type 2) and, to a lesser extent, during insulin resistance, causes oxidative stress. Elevated glucose in diabetes may also react with lipids, resulting in the generation of ROS (45). Diabetes mellitus often includes lipid abnormalities such as elevated LDL-C and cholesterol, and such effects were shown in this in vivo study. These abnormalities may be further exacerbated by the increased oxidizing environment which enhances the formation of oxidized LDLs (oxLDLs), glycated LDL and oxysterols (formed from the oxidation of cholesterol). It has been suggested that these oxidized lipid products can bind to specific receptor proteins or activate inflammatory proteins which generate ROS (45). The import of oxLDLs in the vascular wall is an important mechanism by which ROS and oxidative stress induce atherosclerosis (46). In our study, prooxidant-antioxidant balance was evaluated by measuring MDA and NO levels and enzymatic antioxidants in the diabetic rats. Increased MDA, NO and decreased SOD, GST and CAT activities indicated that the balance changed towards prooxidation in STZ-induced diabetic rats. Safranal treatment of diabetic rats, which restored SOD, GST and CAT activities, may be due to a decrease in free radical generation by safranal and increased 
antioxidant defenses (12). Safranal, a monoterpene aldehyde major constituent of the essential oil of saffron (47), showed good antioxidant activity in vivo $(48,49)$. Safranal administration regulates the expression of antioxidant related genes, and consequently oxidant levels in diabetic animals. In biological systems safranal shows its antioxidant impact via stabilizing membranes (50), inhibiting ROS and reducing peroxidation of unsaturated membrane fatty acids (50). Safranal has been known to function as a radical scavenger inhibiting lipid peroxidation in vivo and in vitro. It also has been reported that safranal supplementation could decrease lipid peroxidation $(47,51)$. Safranal modulates antioxidant gene expression and also upregulates mitochondrial antioxidant genes, leading to a lower mitochondrial oxygen radical generation, which may be responsible at least in part for the improved hyperglycemia, hyperlipidemia and oxidative stress seen in the present study in STZinduced diabetic rats. Additionally, increased SOD, GST and CAT levels after safranal treatment may play an additional role in decreasing oxidative stress. The present study elucidated the antihyperglycemia, hypolipidemic and protective potential of safranal treatment on activities of antioxidant enzymes (SOD, GST, CAT), lipid peroxidation levels and serum NO content in the STZ diabetic rat model.

In summary, the findings in this study show that safranal treatment demonstrates a protective effect in the STZ model of diabetes and its complications by modulation of oxidative stress. Although detailed studies are required for the evaluation of the exact protective mechanism of safranal against diabetic complications in animal models and humans, these in vivo experimental findings demonstrate that safranal exhibits antidiabetic effects in the rat diabetic model by potentiating the antioxidant defense system. The findings support the potential efficacy of safranal for diabetes management.

\section{ACKNOWLEGEMENTS}

The authors would like to thank Research Affairs of Neyshabur University of Medical Sciences for financially supporting this work.

\section{REFERENCES}

1. Chaiyasut C, Kusirisin W, Lailerd N, Lerttrakarnnon P, Suttajit M, Srichairatanakool S. Effects of phenolic compounds of fermented thai indigenous plants on oxidative stress in streptozotocin-induced diabetic rats. J Evid Based Complementary Altern Med, 2011; 3: 1-10.

2. Abou-Seif MA, Youssef AA. Evaluation of some biochemical changes in diabetic patients. Clin Chim Acta, 2004; 346: 161-170.

3. Strain JJ. Disturbances of micronutrient and antioxidant status in diabetes. P Nutr Soc, 1991; 50: 591-604.

4. Baynes JW. Role of oxidative stress in development of complications in diabetes. Diabetes, 1991; 40: 405-412.

5. Mclennan SV, S. Heffernen L. Changes in hepatic glutathione metabolism in diabetes. Diabetes, 1991; 40: 344-348.

6. Mullarkey CJ, D. Edelstein L. Free radical generation by early glycation products: a mechanism for accelerated atherogenesis in diabetes. Biochem Biophys Res Comm, 1990; 173: 932-939.

7. Soto C, R. Recoba H, Barron C, Alvarez L. Silymarin increases antioxidant enzymes in alloxan-induced diabetes in rat pancreas. Comp Biochem Physiol, 2003; 136: 205-212.

8. Young IS, Torney ER. The effect of ascorbate supplementation on 7-oxidative stress in the streptozotocin diabetic rat. Free Radical Biol Med, 2003; 136: 205-212.

9. Abdullaev FJ. Biological effects of saffron. Biofactors, 1993; 4: 83-86.

10. Samarghandian S, Boskabady MH, Davoodi S. Use of in vitro assays to assess the potential antiproliferative and cytotoxic effects of saffron (Crocus sativus L.) in human 6 lung cancer cell line. Pharmacogn Mag, 2010; 6: 309-314

11. Samarghandian S, Tavakkol Afshari J, Davoodi S. Suppression of pulmonary tumor Promotion and induction of apoptosis by Crocus sativus L. Extraction. Appl Biochem Biotechnol, 2011; 164: 238-47.

12. Farahmand SK, Samini F, Samini M, Samarghandian S. Safranal ameliorates antioxidant enzymes and suppresses lipid peroxidation and nitric oxide formation in aged male rat liver. Biogerontology, 2013; 14:63-71.

13. Abdullaev FI. Biomedical properties of saffron and its potential use in cancer therapy and chemoprevention trials. Cancer Det Preven, 2004; 28: 426-432.

14. Abdullaev FI. Use of in vitro assays to assess the potential antigenotoxic and cytotoxic effects of 
saffron (Crocus sativus L.). Toxicol in vitro, 2003; 17: 751-758.

15. Assimopoulou AN, Sinakos Z, Papageorgiou VP. Radical scavenging activity of Crocus sativus L. extract and its bioactive constituents. Phytother Res, 2005; 19: 997-999

16. Kianbakht S, Hajiaghaee R. Anti-hyperglycemic effects of saffron and its active constituents, crocin and safranal, in alloxan-induced diabetic rats. J Med Plants, 2011; 10: 39-43.

17. Bathaie SZ, Mousavi Z. New applications and mechanisms of action of saffron and its important ingredients. Crit Rev Food Sci Nutr, 2010; 50: 761 -786 .

18. Ellman GL. Tissue sulfhydryl groups. Arch Biochem Biophys, 1959; 82: 70-77

19. Genet S, Kale RK, Baquer NZ. Alterations in antioxidantenzymes and oxidative damage in experimental diabetic rattissues: effect of vanadate and fenugreek (Trigonella foe-num graecum). Mol Cell Biochem, 2002; 236: 7-12.

20. Marklund S, Marklund G. Involvement of the superoxide anion radical in the autoxidation of pyrogallol and a convenient assay for superoxide dismutase. Eur J Biochem, 1979; 47: 469-474.

21. Aebi H. Catalase in vitro. Methods Enzymol, 1984; 105: 121-126.

22. Pieper GM, Jordan M, Dondlinger LA, Adams MB, Roza AM. Peroxidative stress in diabetic blood vessels. Reversal by pancreatic islet transplantation. Diabetes, 1995; 44: 884-889

23. Hortelano S, Dewez B, Genaro AM, D'1az-Guerra MJ, Bosca' L. Nitric oxide is released in regenerating liver after partial hepatectomy. Hepatology, 1995; 21: 776-786

24. Bradford MM. A rapid and sensitive method for the quantitation of microgram quantities of protein utilizing the principle of protein dye binding. Anal Biochem, 1976; 72: 248-254.

25. Kianbakht S, Mozaffari K. Effects of saffron and its active constituents, crocin and safranal, on prevention of indomethacin induced gastric ulcers in diabetic and nondiabetic rats. J Med Plants, 2009; 8: 5-8.

26. Rahbani M, Mohajeri D, Rezaie A, Doustar Y, Nazeri M. Attenuation of oxidative stress of hepatic tissue by ethanolic extract of saffron (dried stigmas of Crocus sativus L.) in streptozotocin (STZ)induced diabetic rats. Afr J Pharm Pharmacol, 2011; 5: 2166-2173.

27. Kamalipour M, Akhondzadeh S. Cardiovascular Effects of Saffron: An Evidence-Based Review J Teh Univ Heart Ctr, 2011; 2: 59-61.

28. Budin S, Othman F, Louis S, A. Bakar M, Das S, Mohamed J. The effects of palm oil to cotrienolrich fraction supplementation on biochemical parameters, oxidative stress and the vascular wall of streptozotocin-induced diabetic rats. Clincs, 2009; 64: 235-244.

29. Yazdanparast R, Ardestani A, Jamshidi S. Experimental diabetes treated with Achillea santolina: Effect on pancreatic oxidative parameters. J Ethnopharmacol, 2007; 112: 13-18.

30. Elsner M, Guldbakke B, Tiedge M, Munday R, Lenzen S. Relative importance of transport and alkylation for pancreatic -cell toxicity of streptozotocin. Diabetologia, 2000; 43: 1528-1533.

31. Szkudelski T. The mechanism of alloxan and streptozotocin action in cells of the rat pancreas. Physiol Res, 2001; 50: 537-546.

32. Turk J, Corbett JA, Ramanadham S, Bohrer A, McDaniel ML. Biochemical evidence for nitric oxide formation from streptozotocin in isolated pancreatic islets. Biochem Biophys Res Commun, 1993; 197: 1458-1464.

33. Kroncke KD, Fehsel K, Sommer A, Rodriguez ML. Kolb- Bachofen, V. Nitric oxide generation during cellular metabolization of the diabetogenic Nmethyl-N-nitroso-urea streptozotozin contributes to islet cell DNA damage. Biol Chem Hoppe Seyler, 1995; 376: 179-185.

34. Chatzigeorgiou A, Halapas A, Kalafatakis K, Kamper E. The use of animal models in the study of diabetes mellitus. In Vivo, 2009; 3: 245-258.

35. Shahady E. Hyperlipidemia in diabetes-etiology, consequences and treatment Adapted from Libby P. Circulation, 1995; 91: 2844-2850.

36. Grune T, Siems WG, Petras T. Identification of metabolic pathways of the lipid peroxidation product 4-hydroxynonenal in in situ perfused rat kidney. J Lipid Res, 1997; 38: 1660-1665.

37. Chalupsky K, Cai H. Endothelial dihydrofolate reductase: critical for nitric oxide bioavailability and role in angiotensin II uncoupling of endothelial nitric oxide synthase. Proc Natl Acad Sci USA, 2005; 102: 9056-9061.

38. Guzik TJ, Mussa S, Gastaldi D. Mechanisms of increased vascular superoxide production in human diabetes mellitus: role of NAD (P) $\mathrm{H}$ oxidase and endothelial nitric oxide synthase. Circulation, 2002; 105: 1656-1662.

39. Bardell AL, MacLeod KM. Evidence for inducible nitric-oxide synthase expression and activity in vascular smooth muscle of streptozotocin-diabetic rats. J Pharmacol Exp Ther, 2001; 296: 252-259

40. Soto CP, Perez BL, Favari LP, Reyes JL. Prevention of alloxan-induced diabetes mellitus in the rat by silymarin. Comp Biochem Physiol C Pharmacol Toxicol Endocrinol, 1998; 119: 125129.

41. El-Missiry MA. Enhanced testicular antioxidant system by ascorbic acid in alloxan diabetic rats. 
Comp Biochem Physiol C Pharmacol Toxicol Endocrinol, 1999; 124: 233-237.

42. Nishikimi M, Rao NA, Yagl K. The occurrence of superoxide anion in the reaction of reduced phenazine methosulphate and molecular oxygen. Biochem Biophys Res Commun, 1972; 46: 844853

43. Arasteh A, Aliyev A, Khamnei S, Delazar A, Mesgari M, Mehmannavaz Y. Effects of hydromethanolic extract of saffron (Crocus sativus) on serum glucose, insulin and cholesterol levels in healthy male rats. J Med Plants Res, 2010; 4: 397402.

44. Xiang M, Yang M, Zhou C, Liu J, Li W, Qian Z. Crocetin prevents AGEs-induced vascular endothelial cell apoptosis. Pharmacol Res, 2006; 54: 268-274.

45. Johansen JS, Harris AK, Rychly DJ, Ergul A.Oxidative stress and the use of antioxidants in diabetes: linking basic science to clinical practice. Cardiovasc Diabetol, 2005; 4:5.

46. Wiernsperger NF.Oxidative stress as a therapeutic target in diabetes: revisiting the controversy. Diabetes Metab, 2003; 29: 579-85.
47. Assimopoulou AN, Sinakos Z, Papageorgiou VP. Radical scavenging activity of Crocus sativus L. extract and its bioactive constituents. Phytother Res, 2005; 19: 997-1000.

48. Bharti S, Golechha M, Kumari S, Siddiqui KM, Arya DS. Akt/GSK-3b/eNOS phosphorylation arbitrates safranal- induced myocardial protection against ischemia-reperfusion injury in rats. Eur $\mathbf{J}$ Nutr, 2012; 51: 719-727.

49. Halataei BA, Khosravi M, Arbabian S, Sahraei H, Golmanesh L, Zardooz H, Jalili C, Ghoshooni H. Saffron (Crocus sativus) aqueous extract and its constituent crocin reduces stress-induced anorexia in mice. Phytother Res, 2011; 25: 1833-1838.

50. Hosseinzadeh H, Sadeghnia HR. Safranal, a constituent of Crocus sativus (saffron), attenuated cerebral ischemia induced oxidative damage in rat hippocampus. J Pharm Pharm Sci, 2005; 8:394-399.

51. Papandreou MA, Tsachaki M, Efthimiopoulos S, Cordopatis P, Lamari FN, Margarity M. Memory enhancing effects of saffron in aged mice are correlated with antioxidant protection. Behav Brain Res, 2011; 219: 197-20. 